\title{
Sekundärer Hypogonadismus: Testosteronsubstitution ist wirksam und sicher
}

— Rund $10 \%$ aller Männer haben nach aktuellen Daten aus Deutschland deutlich erniedrigte Testosteronwerte. Im Rahmen einer Screening-Aktion der Stiftung "Mann und Gesundheit" wurden bei 2.982 Männern ab 18 Jahren, die im Mittel 54 Jahre alt waren, die Testosteronspiegel gemessen, berichtete Dr. Tobias Jäger, Essen. 9 \% aller Studienteilnehmer hatten deutlich erniedrigte Testosteronwerte $(<2,5 \mathrm{ng} / \mathrm{ml})$, bei weiteren $18 \%$ lagen die Werte im kritischen Bereich zwischen 2,5 und $3,5 \mathrm{ng} / \mathrm{ml}$, bei denen durchaus bereits klinische Symptome auftreten könnten. Erste Symptome sind in der Regel Vitalitäts- und Libidoverlust, später kommen Gewichtszunahme, Schlaf- und Konzentrationsstörungen, Depressionen, Metabolisches Syndrom und Diabetes hinzu.
Unter einer Testosteronsubstitution bessern sich viele Beschwerden, wie Daten der IPASS (International, multi-centre, Post-Authorisation Surveillance Study on the use of $\mathrm{Ne}$ bido $^{\oplus}$ to assess tolerability and treatment outcomes in daily clinical practice)-Studie belegen. 1.438 hypogonadale Männer aus 23 Ländern wurden in dieser Studie mit der Drei-Monats-Spritze Nebido ${ }^{\circledR}$ behandelt, berichtete Prof. Michael Zitzmann, Münster. Bereits nach zwei Injektionen hatte sich die Stimmungslage der Männer deutlich gebessert, nach fünf Injektionen (40-44 Wochen) gaben drei Viertel der Männer eine positive Stimmung an im Vergleich zu nur einem Fünftel bei Studienbeginn. Der Bauchumfang der Männer verringerte sich deutlich und bei Studienteilnehmern mit Diabetes sank der HbA1c um 1,1\% [Zitzmann $\mathrm{M}$ et al., J Sex Medicine 2012 epub].,Das liegt im Bereich eines modernen Antidiabetikums", sagte Zitzmann. Laut ersten Daten der "Seattle-Veteranen-Studie Teil 2" korreliert eine Testosteronsubstitution bei hypogonadalen Männern auch mit einer verringerten Mortalität [Shores et al., JCEM 2012 epub]. Und die Therapie war sicher, betonte Zitzmann: Im Verlauf von bisher vier Jahren ist die Inzidenz von Prostatakarzinomen in der behandelten und unbehandelten Gruppe auf gleichem Niveau ( $1,6 \%$ vs. $2,0 \%$ ).

Roland Fath

Pressegespräch "Aktuelles zur Männergesundheit - Aus Forschung und Praxis", Leipzig, im Rahmen des 64. Kongresses der Deutschen Gesellschaft für Urologie, Leipzig, 27. September 2012;

Veranstalter: Jenapharm, Jena

\section{Prostatakarzinom:}

\section{Neuer Therapiebegleiter unterstützt Arzt und Patient}

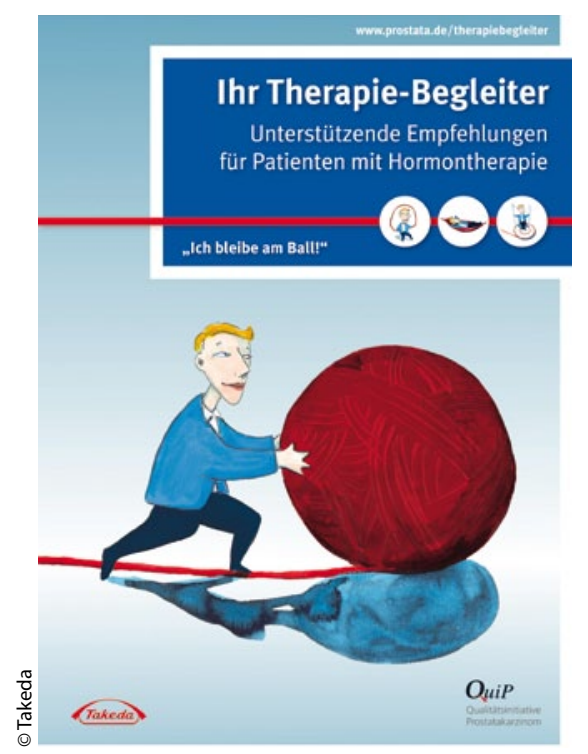

Informationen und Empfehlungen für Patienten unter Hormontherapie

- Patienten mit Prostatakarzinom (PCa) benötigen eine intensive Beratung durch ihren behandelnden Arzt, vor allem wenn eine antihormonelle Therapie vorgesehen ist. Viele PCa-Patienten fragen sich, was sie neben der ärztlich verordneten Therapie noch tun können, um aktiv an ihrer Krankheitsbewältigung mitzuwirken und die Lebensqualität zu verbessern. Takeda hat deshalb in Zusammenarbeit mit Urologen, Psychoonkologen, Sport- und Ernährungsmedizinern den Ratgeber "Ich bleibe am Ball“ entwickelt, der über den Außendienst-Mitarbeiter bezogen werden kann.

Das Informationspaket enthält einen Leitfaden zurTherapiebegleitung und Bewegung, eine Entspannungs- $C D$, Tipps für eine ausgewogene Ernährung sowie konkrete sportliche Übungsvorschläge. Der Ratgeber richtet sich vor allem an Patienten, die sich einer hormonablativen Behandlung unterziehen müssen. Die Androgendeprivation gehört seit vielen Jahren zum Therapiestandard bei fortgeschrittenem PCa, ist aber auch mit Nebenwirkungen assoziert, so Prof. Thomas Ebert, Fürth. Es kann dadurch in der Folge zu Bluthochdruck, Osteoporose oder Veränderungen des Zucker- und Fettstoffwechsels kommen. „Aber es gibt Möglichkeiten, mit denen man gegensteuern kann“, ist Ebert überzeugt.

Dass körperliche Aktivität das physische und psychische Befinden onkologischer Pati- enten verbessern kann, betonte auch Dr. FreerkT. Baumann, Köln. Regelmäßiges Ausdauer-, Kraft- und Koordinationstraining wirke nicht nur Antriebslosigkeit, Muskelund Knochschwund entgegen, sondern könne auch neues Selbstvertrauen vermitteln. „Wir haben das Programm so aufgebaut, dass es motiviert. Es müssen einfache Trainingseinheiten sein, die den Patienten nicht unter Leistungsdruck setzen." Nur wer Freude an der Bewegung hat, bleibe auch „längerfristig am Ball".

Nützliche Tipps erhalten Patienten auch im Internet unter www.prostata.de/therapie begleiter. Dr. Martina-Jasmin Utzt
Pressegespräch „Ich bleibe am Ball“ Therapiebegleitung für Patienten mit einer Hormontherapie beim Prostatakarzinom, im Rahmen des 64. Kongresses der Deutschen Gesellschaft für Urologie, Leipzig, 27. September 2012; Veranstalter:Takeda, Aachen 